\title{
PROSPECTIVA ESTRATÉGICA DE MICHEL GODET: UM MAPEAMENTO SISTEMÁTICO DO MÉTODO DOS CENÁRIOS NA BASE SCOPUS
}

\author{
Laís Sant'Anna Fonseca \\ Universidade Estadual do Norte Fluminense Darcy Ribeiro - UENF \\ Av. Alberto Lamego, 2000, Parque Califórnia, Campos dos Goytacazes/RJ - CEP: 28013-602 \\ laissfon@pq.uenf.br \\ Edson Terra Azevedo Filho \\ Universidade Estadual do Norte Fluminense Darcy Ribeiro - UENF \\ Av. Alberto Lamego, 2000, Parque Califórnia, Campos dos Goytacazes/RJ - CEP: 28013-602 \\ edsonterrafilho@gmail.com \\ Cristiano Manhães de Oliveira \\ Universidade Estadual do Norte Fluminense Darcy Ribeiro - UENF \\ Av. Alberto Lamego, 2000, Parque Califórnia, Campos dos Goytacazes/RJ - CEP: 28013-602 \\ cristianomoliveira@gmail.com
}

\section{RESUMO}

Atualmente, vive-se numa época globalizada e dinâmica, onde as metodologias prospectivas oferecem suporte para nortear o futuro, apresentando possibilidades alcançáveis e desejadas. Contudo, os métodos prospectivos oferecem ferramentas que concedem flexibilidade estratégica para a tomada de decisões, a partir da oportunidade da ação. Dentre as duas correntes principais, destaca-se a corrente francesa, em função de uma maior valorização do aspecto humano, tendo como um dos principais expoentes Michel Godet, que propõe o método dos cenários. Este se baseia na identificação e projeção das variáveis-chaves influentes em determinado contexto, assim como a relação entre os atores, até a efetiva projeção de cenários para a realização do exercício prospectivo. Este método pode ser dividido em dois grandes blocos compostos pela construção da base analítica e histórica e a efetiva elaboração dos cenários. O método de Godet possui cinco ferramentas MICMAC, MACTOR, SMIC, MORPHOL e MULTIPOL - podendo ser aplicado de forma completa ou compartimentada em quaisquer setores e temáticas. O intuito deste trabalho é aprofundar os conhecimentos acerca das aplicações do Método dos cenários nas áreas da engenharia presente no meio científico para investigar e entender os diferentes ramos e contextos que aplicaram as respectivas ferramentas usadas no método. Deste modo, define-se como objetivo geral a elaboração do mapeamento sistemático, cobrindo todo o período de publicações e citações na base de dados Scopus. A metodologia aplicada foi o mapeamento sistemático, alicerçado na revisão sistemática, com o apoio de índices bibliométricos e gráficos com o intuito de aprofundar as peculiaridades qualitativas e quantitativas. O mapeamento sistemático realiza um estudo mais amplo da revisão sistemática, identificando quais evidências estão disponíveis e as lacunas para direcionar o próximo foco. Este mapeamento exibe um resumo visual dos resultados obtidos que além de oferecer repetibilidade ao processo, reduz eventuais vieses que possam aparecer nas pesquisas. $\mathrm{O}$ mapeamento sistemático foi realizado utilizando-se a base de dados Scopus, com o acesso até 24 de maio de 2019, utilizando as condições de article title, abstract e keywords,com o tipo de documento article, obtendo-se 70 registros, no qual apenas 24 artigos foram selecionados pelo enquadramento no escopo da pesquisa. Dentre estes artigos qualificados, 4 são teóricos e 20 são práticos e a ferramenta mais utilizada foi a MICMAC que faz a análise estrutural de variáveis. $\mathrm{O}$ 
presente artigo tem como finalidade a apresentação das informações sobre o método dos cenários e a aplicação de suas ferramentas. Os resultados obtidos através do termo pesquisado sugerem que os artigos não utilizam todas as ferramentas, o que é justificado pela adaptabilidade para sua aplicação em determinados casos, a fim de obter uma cobertura mais consistente e ampla. O objetivo proposto foi atendido ao apresentar um estudo compilando quais anos, autores, instituições, países, áreas e palavras-chave mais relacionados com o método dos cenários. Espera-se com a realização desse artigo científico o estímulo na utilização do método por outros pesquisadores, a partir de uma maior clarificação das possibilidades de aplicação.

Palavra-chave: Mapeamento sistemático; Revisão Sistemática; Cenários prospectivos; Método dos cenários; Godet.

\begin{abstract}
Today we live in a globalized and dynamic era, where prospective methodologies provide support to guide the future, presenting achievable and desired possibilities. However, prospective methods offer tools that give strategic decision-making flexibility from the opportunity of action. Among the two main currents, the French current stands out, due to a greater appreciation of the human aspect, having as one of the main exponents Michel Godet, who proposes the scenario method. This is based on the identification and projection of key influential variables in a given context, as well as the relationship between the actors, until the actual projection of scenarios for the prospective exercise. This method can be divided into two major blocks composed by the construction of the analytical and historical basis and the effective elaboration of the scenarios. Godet's method has five tools MICMAC, MACTOR, SMIC, MORPHOL and MULTIPOL - can be applied completely or compartmentalized in any sectors and themes. The aim of this work is to deepen the knowledge about the applications of the Scenario Method in the fields of engineering in the scientific field to investigate and understand the different branches and contexts that applied the respective tools used in the method. Thus, the general objective is the elaboration of systematic mapping, covering the entire period of publications and citations in the Scopus database. The methodology applied was the systematic mapping, based on the systematic review, supported by bibliometric indexes and graphs in order to deepen the qualitative and quantitative peculiarities. Systematic mapping conducts a broader study of the systematic review, identifying what evidence is available and the gaps to direct the next focus. This mapping shows a visual summary of the results obtained that, besides offering repeatability to the process, reduces any biases that may appear in the research. Systematic mapping was performed using the Scopus database, accessed until May 24, 2019, using the conditions of article title, abstract and keywords, with document type article, obtaining 70 records, in which Only 24 articles were selected according to the scope of the research. Among these qualified articles, 4 are theoretical and 20 are practical and the most used tool was the MICMAC that makes the structural analysis of variables. The purpose of this article is to present information about the scenario method and the application of its tools. The results obtained through the search term suggest that the articles do not use all the tools, which is justified by the adaptability to their application in certain cases, in order to obtain a more consistent and broad coverage. The proposed objective was met by presenting a study compiling which years, authors, institutions, countries, areas and keywords most related to the scenario method. It is expected with the accomplishment of this scientific article the stimulus in the use of the method by other researchers, from a greater clarification of the possibilities of application.
\end{abstract}

Keywords: Systematic mapping; Systematic review; Prospective scenarios; Scenario method; Godet. 


\section{Como Citar:}

FONSECA, L. S.; AZEVEDO FILHO, E. T.; OLIVEIRA, C. M. Prospectiva Estratégica de Michel Godet: Um mapeamento sistemático do método dos cenários na base Scopus. In: SIMPÓSIO DE PESQUISA OPERACIONAL E LOGÍSTICA DA MARINHA, 2019, Rio de Janeiro, RJ. Anais SPOLM 2019. Rio de Janeiro: Centro de Análises de Sistemas Navais, 2019.

\section{INTRODUÇÃO}

$\mathrm{O}$ homem desde sua origem se antecipa proativamente às incertezas para se capacitar na tomada de decisões. Os métodos prospectivos são ferramentas que propiciam a flexibilidade estratégica para o auxílio de escolhas sobre o futuro, alcançando a oportunidade da ação ao invés da aceitação (GODET, 1993).

Na definição de Porter: "cenários são visões parciais e internamente consistentes de como o mundo será no futuro e que podem ser escolhidas de modo a limitar o conjunto de circunstâncias que podem vir a ocorrer" (PORTER, 1985).

O planejamento dos cenários admite a existência de uma incerteza. Isso, inserido na cultura organizacional, proporciona aos gestores um diferencial estratégico que, além de reduzir os riscos na tomada de decisão e aumentar a probabilidade de acerto considerando essas mudanças inevitáveis, permite guiar a empresa com maior objetividade na manutenção do seu negócio, pois disponibiliza melhores condições de competitividade e adaptação (RODRIGUES; ROJO; BERTOLINI, 2013).

$\mathrm{Na}$ trajetória teórica das metodologias e ferramentas da prospectiva estratégica, destacam-se duas correntes que possuem características distintas. A primeira é conhecida como escola francesa ou La Prospective, foi fundada na França em meados da década de 60 por Bertrand de Jouvenel. Esta corrente possui trabalhos sobre as questões futuras e supõe na criação e mudança do futuro de modo proposital pelos atores sociais (AZEVEDO FILHO, 2015).

A segunda corrente originou-se nos Estados Unidos, no fim da década de 60 e possui como principais autores Herman Khan e Peter Schwartz. Ao longo da Segunda Guerra Mundial, foram criados grupos com o intuito da pesquisa tecnológica estratégica para aperfeiçoar a segurança. Esta possui tradições inglesa, alemã e americana, conhecida como corrente anglo-saxônica e usa a tecnologia como principal propulsor da mudança socioeconômica (SOUZA, 2009).

As diferenças entre ambas as escolas são a natureza da elaboração dos cenários das abordagens. A escola francesa de Michel Godet valoriza o processo formal ao elaborar os cenários, mesmo incorporando aspectos intuitivos, há etapas bem definidas a serem atingidas até cumprir os cenários finais. $\mathrm{O}$ método possui como limitação o longo percurso de todas as etapas que pode ser demorado e correr o risco de não ser percorrido até o final e, há casos de agilidade no planejamento (GODET, 1991).

Apesar da prospectiva estar centralizado neste artigo, abordam-se aspectos inevitáveis na estruturação dos atores com a proposta de enfrentar os desafios futuros. Portanto, optou-se por usar uma pesquisa da corrente francesa, em função da valorização do aspecto humano nos estudos prospectivos que abordam questões sobre o futuro da sociedade em variadas dimensões como social, econômica e tecnológica (SARAGOÇA, 2012).

Estabelecendo-se como tema do artigo a La Prospective de Michel Godet, uma das aplicações que possuem destaque é o método dos cenários que de acordo com Godet (1993), um cenário é um conjunto formado pela descrição de uma situação futura e do encaminhamento dos acontecimentos que permitem passar da situação de origem a essa situação futura, visando sempre a ação e não a predeterminação.

Nas pesquisas realizadas na base de dados Scopus sobre o autor Michel Godet, 
pode-se conhecer sua representatividade acadêmica, apresentando 46 artigos de própria autoria ou coautoria e 942 citações em 747 artigos de outros pesquisadores no período de 1976 até 2019, com expressivo aumento nos últimos dez anos.

Como o método dos cenários proposto por Godet, possui uma vasta área de atuação, para compreender melhor a importância, estabeleceu-se o seguinte questionamento: qual a relevância acadêmica do método dos cenários e quais são as suas principais aplicações?

A fim de cumprir o nosso objetivo, realizou um mapeamento sistemático da literatura para investigar o panorama das aplicações do método dos cenários de Michel Godet. Além do objetivo proposto, este artigo científico possui como propósito o estímulo no uso do método por outros pesquisadores, devido a clarificação das possíveis aplicações.

$\mathrm{O}$ artigo inicia-se com esta introdução e, na seção 2, é descrito o conceito de método dos cenários que será utilizado neste artigo. A seção 3 descreve a metodologia de pesquisa aplicado neste trabalho; a seção 4 apresenta os resultados obtidos da pesquisa bibliométrica; a seção 5 apresenta as conclusões; a seção 6 são apresentadas as referências utilizadas no trabalho.

\section{MÉTODO DOS CENÁRIOS}

Antes da apresentação do esquema integrado é conveniente destacar a definição e as origens do método dos cenários. Um cenário é um conjunto formado pela descrição de uma situação futura e do encaminhamento dos acontecimentos que permitem passar da situação de origem a essa situação futura. A palavra cenário é frequentemente utilizada de forma abusiva para qualificar um qualquer jogo de hipóteses. Recordemos que as hipóteses de um cenário devem preencher simultaneamente cinco condições: pertinência, coerência, verossimilhança, importância e transparência.

Distinguem-se dois tipos de cenários: exploratórios que partem das tendências passadas e presentes e conduzem a futuros aceitáveis; e normativos, ou de antecipação que são construídos a partir de imagens alternativas do futuro desejados ou temidos; são concebidos de forma retrospectiva. Os cenários exploratórios ou de antecipação podem, além disso, levar em consideração as evoluções prováveis ou extremas, com tendenciais ou contrastados.

Dentre os métodos de análise prospectiva estratégica, um dos que merece destaque é o método dos cenários, pois apesar da elaboração de cenários e análise prospectiva não sejam semelhantes, a construção de cenários constantemente assume um papel central em grande parte dos estudos prospectivos (GODET; DURANCE; DIAS, 2008).

Para Godet (2000), não existe um único método quando se desenvolve cenários, mas infinitos métodos que propiciam sua construção, sendo alguns mais simples e outros mais elaborados. Contudo, esse autor afirma que existe consenso na aplicação do método de cenários apenas para uma abordagem que contenha um número de etapas específicas interrelacionadas: análise de sistemas, retrospectiva, estratégia dos atores e elaboração de cenários.

A descrição dos cenários estudados é baseada na provável evolução das variáveischave e nas hipóteses de comportamento dos atores (SUTTER, 2012). O método dos cenários proposto por Godet (Figura 1) organiza o exercício prospectivo com o intuito de definir as estratégias e esclarecer os meios de execução, dividindo em duas etapas:

Construção da base: representa atualmente o estado do sistema, incluindo as variáveis-chave e o conjunto de atores. Segundo Godet et al. (2008), recomendam que esta fase seja produzida essencialmente em workshops que permitam um levantamento aprofundado. Com isso, nesta etapa as variáveis são definidas, classificadas e as suas interações são analisadas pela matriz. Por fim, realiza a análise dos atores com as variáveis- 
chave.

Construção de cenários: resulta no estudo das incertezas. Pelo método de Godet, é feita uma análise morfológica das variáveis e dos fatos futuros mais importantes e que podem alcançar um grande número de potenciais cenários. Para a análise de probabilidade de cada combinação, usa-se o software SMIC, que receberá o resultado da consulta a especialistas quanto às probabilidades de ocorrência de cada combinação. Para cada cenário designado, deve-se construir uma sequência, partindo da situação atual até a visão do futuro determinada pelo cenário escolhido.

Figura 1 - Etapas do método dos cenários

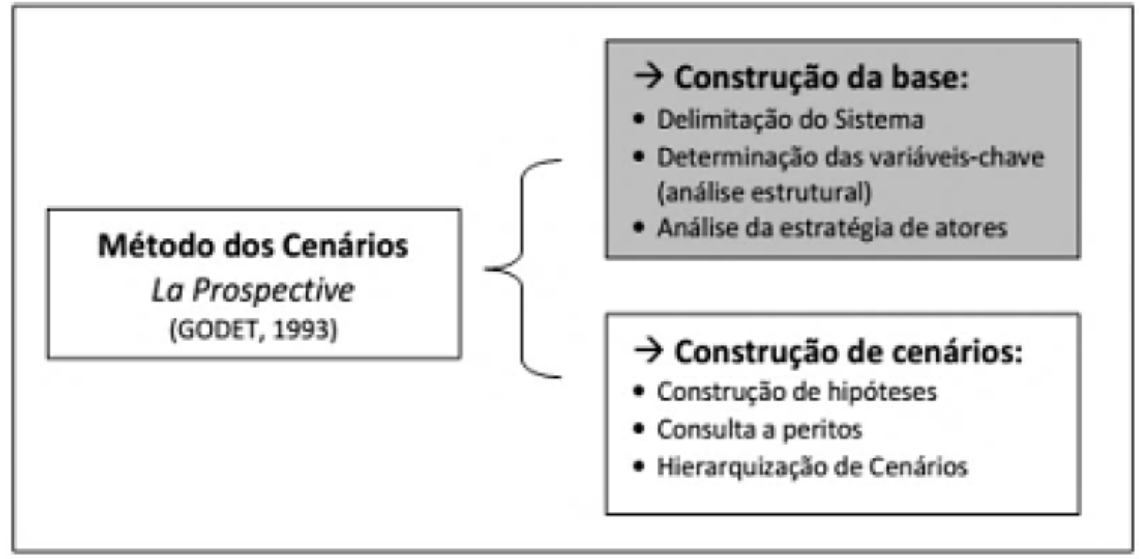

Fonte: Adaptado de Godet (1993)

O método dos cenários engloba cinco ferramentas - MICMAC, MACTOR, SMIC, MORPHOL e MULTIPOL - com a aplicação de forma completa ou compartimentada em quaisquer setores e temáticas, conforme apresentado na Figura 2.

Figura 2 - Método dos cenários

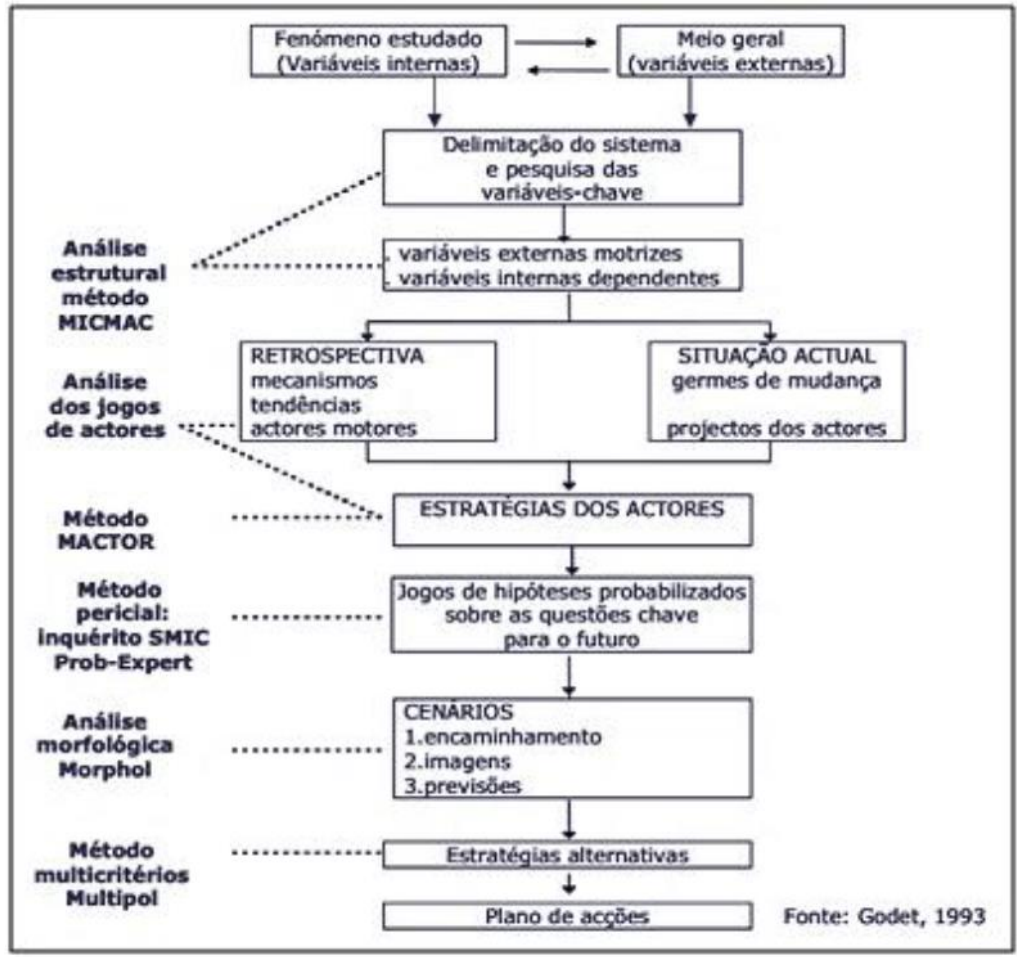

Fonte: Godet (1993) 


\section{METODOLOGIA}

Atualmente, a comunidade científica mundial tem publicado grande número de artigos em diferentes campos científicos. Neste ambiente, é essencial saber quais bases de dados são eficientes e objetivas para pesquisas de literatura. As duas bases de dados mais abrangentes são Web of Science e Scopus. Além de pesquisar a literatura, estes dois bancos de dados são usados para classificar periódicos em termos de sua produtividade e as citações totais recebidos para indicar o impacto de revistas, prestígio ou influência (CHADEGANI; SALEHI; YUNUS; EBRAHIM, 2013).

A organização do conjunto de softwares especializados em dados globais e que são particularmente adequados para análise de várias etapas, usam diferentes tipos de ferramentas de software. Este último é particularmente relevante, uma vez que permite a integração de uma série de pacotes $\mathrm{R}$ recentemente desenvolvidos especificamente para bibliometria (GULER, 2016). Neste artigo, utilizaremos as bibliotecas bibliometrix para obtenção de alguns resultados na seção 4.

O biblioshiny é um aplicativo que fornece uma interface web para bibliometrix. O pacote bibliometrix fornece um conjunto de ferramentas para pesquisa quantitativa em bibliometria e cienciometria. Esta é uma ferramenta de código aberto para a execução de uma análise abrangente de mapeamento da literatura científica (ARIA; CUCCURULLO, 2017).

Foi programado em linguagem $\mathrm{R}$ para ser flexível e facilitar a integração com outros pacotes estatísticos e gráficos. De fato, a bibliometria é uma ciência em constante mudança e a bibliometrix tem a flexibilidade de ser rapidamente atualizada e integrada. Seu desenvolvimento pode abordar uma comunidade grande e ativa de desenvolvedores formada por pesquisadores proeminentes (CUCCURULLO; ARIA; SARTO, 2016).

A bibliometrix fornece várias rotinas para importar dados bibliográficos do Scopus, das bases de dados Web of Science, PubMed e Cochrane do Clarivate Analytics, realizando análises bibliométricas e construindo matrizes de dados para co-citação, acoplamento, análise de colaboração científica e análise conjunta.

A revisão sistemática da literatura trata-se de uma metodologia rigorosa proposta para identificar os estudos sobre um tema em questão a partir da aplicação de métodos sistematizados de busca visando avaliar a qualidade e validade dos referidos estudos, assim como sua aplicabilidade. A revisão sistemática é realizada baseada em critérios predefinidos que são utilizados para evitar os possíveis vieses e possibilitar uma análise mais objetiva dos resultados, facilitando uma síntese conclusiva (SCHÜTZ; SANT'ANA; SANTOS, 2011).

No entanto, a revisão sistemática também tem vários inconvenientes, sendo o principal deles que exigem um esforço considerável. Em engenharia de software, as revisões sistemáticas concentraram-se em estudos quantitativos e empíricos, mas um grande conjunto de métodos são usados para sintetizar a existência de resultados de pesquisas qualitativas (DIXON-WOODS et al., 2005).

Um tipo de revisão complementar à revisão sistemática é o de estudo de mapeamento sistemático, que segundo Kitchenham (2007), é uma revisão ampla de estudos primários numa área específica que busca identificar que evidências estão disponíveis nessa área. De acordo com Petersen et al. (2008), afirma que o mapeamento sistemático é um método, cujo objetivo é construir um esquema de classificação e estrutura em um campo de interesse.

Embora, um mapeamento sistemático estudo e uma revisão sistemática da literatura compartilham algumas semelhanças (por exemplo, no que diz respeito à busca e seleção de estudo), eles são diferentes em termos de metas e, portanto, abordagens para análise de dados. Enquanto revisões sistemáticas objetivam sintetizar evidências, a força da evidência, 
mapas sistemáticos são primariamente preocupados com a estruturação de uma área de pesquisa (PETERSEN, 2015).

Estudos de mapeamento sistemático são usados para estruturar uma área de pesquisa, enquanto revisões sistemáticas são focadas na coleta e síntese de evidências (PETERSEN, 2015). O estudo de mapeamento sistemático fornece uma estrutura do tipo de relatórios de pesquisa e resultados que foram publicados através de categorias. Normalmente é fornecido por um sumário visual, o mapa de seus resultados (PETERSEN et al., 2008).

Isso geralmente requer menos esforço, ao mesmo tempo em que oferece uma visão geral mais detalhada. Anteriormente, estudos sistemáticos de mapeamento em engenharia de software foram fortemente recomendados para áreas de pesquisa onde há falta de estudos primários relevantes de alta qualidade (KITCHENHAM; CHARTERS, 2007).

Com o mapeamento sistemático bem especificado, é possível desconsiderar algumas etapas na revisão sistemática. Principalmente, a etapa de identificação de necessidade da revisão e a etapa de identificação de pesquisas primárias e pesquisas similares. Em vista disso, espera-se ganhar tempo na realização das revisões sistemáticas e reduzir o esforço necessário conforme mostrado na Figura 3.

Figura 3 - Etapas do processo de pesquisa

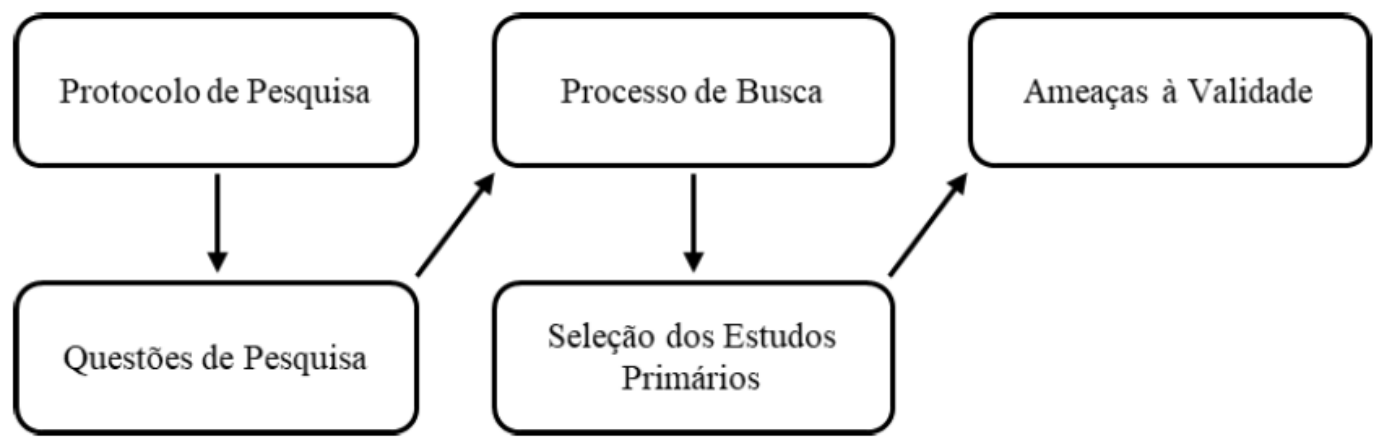

Fonte: Adaptado de Kitchenham (2007)

\subsection{PROTOCOLO DE PESQUISA}

O presente mapeamento sistemático de literatura seguiu os procedimentos propostos por Kitchenham e Charters (2007), conforme a seguir:

Estágio 1 - Planejamento: identificação da necessidade do mapeamento; especificação da (s) pergunta (s) de pesquisa; e desenvolvimento de um protocolo de mapeamento;

Estágio 2 - Realização: identificação de pesquisa; seleção de estudos primários; extração de dados; e síntese de dados;

Estágio 3 - Relato: especificação dos mecanismos de divulgação e formatação do relatório principal.

\subsection{QUESTÕES DE PESQUISA}

O principal objetivo de um mapeamento sistemático é fornecer uma visão geral de uma área de pesquisa e identificar a quantidade e o tipo de pesquisa e resultados disponíveis (PETERSEN, 2008). A especificação das questões de pesquisa é o ponto de partida para qualquer mapeamento sistemático (KITCHENHAM; CHARTERS, 2007). Desta forma, esta pesquisa buscou responder às seguintes questões:

QP1: Quais são os autores e anos de publicação que usam o método dos cenários proposto por Godet? 
QP2: Qual a frequência de publicações em periódicos da base Scopus referentes ao método dos cenários?

QP3: Quais os periódicos mais citados nesse método?

QP4: Quais são os países que são destaques na utilização dessa metodologia de cenários?

QP5: Quais são os países mais citados nesse método dos cenários?

QP6: Quais são as palavras-chave mais utilizadas áreas nesse método?

QP7: Quais os termos mais usados nos títulos e resumos nesse método?

\subsection{PROCESSO DE BUSCA}

O rigor no processo de busca diferencia mapeamentos sistemáticos de outros tipos de revisões (KITCHENHAM; CHARTERS, 2007). Mapeamento e revisões sistemáticas são diferentes em termos de objetivos, questões de validade e implicações. Assim, eles devem ser usados de forma complementar e exigem métodos diferentes (PETERSEN, 2008). A estratégia para identificar as pesquisas publicadas implica na determinação das fontes de busca, que no caso foi utilizada a base Scopus.

A Scopus é uma base de dados multidisciplinar com citações e resumos da literatura, revistas, jornais comerciais, livros, registros de patentes e publicações de conferências. Este fornece ferramentas para rastreamento, análise e visualização de resultados de pesquisa. É o maior banco de dados de citações com mais de 21.500 títulos de mais de 5.000 editores internacionais e oferece a visão mais abrangente da produção mundial de pesquisa nos campos da ciência, tecnologia, medicina, ciências sociais e artes e humanidades (JOSHI, 2016).

A string de busca, contendo sinônimos relevantes, foi desenvolvida a seguir:

( TITLE-ABS-KEY ( foresight OR prospective ) AND TITLE-ABS-KEY ("Scenario* Analysis" OR "scenario* design" OR "scenario* planning" OR "scenario* building" OR "scenario* method" ) AND REF ( godet ) )

\subsection{SELEÇÃO DOS ESTUDOS PRIMÁRIOS}

Os estudos primários relevantes já obtidos, precisam ser avaliados e para isso, é necessário definir alguns critérios de inclusão e exclusão. Estes critérios identificam os estudos primários que tem evidência direta na questão de pesquisa (KITCHENHAM; CHARTERS, 2007).

Para a obtenção dos resultados coerentes, os critérios de inclusão e exclusão foram definidos baseados nas questões de pesquisa. Os critérios mostram que as questões de pesquisa influenciaram critérios de inclusão e exclusão, assim, a parte empírica é considerada apenas para o design do mapa. É útil excluir os artigos que apenas mencionaram o foco principal em frases introdutórias no resumo.

Inclusão: trabalhos que são artigos de periódicos; estudos nas línguas: espanhol, francês, inglês e português.

Exclusão: estudos duplicados e estudos que apenas citam o método dos cenários e não o aplicam, nem de forma prática nem teórica.

O processo de seleção foi dividido em duas etapas distintas:

Etapa 1: leitura dos títulos, palavras-chave, resumo e conclusão;

Etapa 2: os estudos incluídos são documentados. Cada estudo é lido por completo e através de formulários é realizada a extração dos dados.

\subsection{AMEAÇAS À VALIDADE}

$\mathrm{O}$ protocolo de mapeamento segue passos para garantir a pesquisa mais correta e objetiva. Porém, foram identificadas limitações ao longo do estudo. 
Percebeu-se que em relação à identificação dos artigos publicados, a string de busca pode não selecionar todos os sinônimos existentes para o termo cenários prospectivos e, assim, ser insuficiente para alcançar todos os estudos da área.

Já o processo de busca foi realizado na base de dados Scopus, portanto alguns estudos podem não estar publicados e, consequentemente, ficarem de fora da pesquisa.

\section{RESULTADO}

Nesta seção são apresentados os resultados e as discussões sobre o mapeamento sistemático efetuado. Contudo, apresentará o processo de seleção de dados, extração de dados e a apresentação das respostas às questões da pesquisa.

\subsection{PROCESSO DE SELEÇÃO E EXTRAÇÃO DE DADOS}

Neste tópico, o processo de seleção dos trabalhos foi realizado em duas etapas. $\mathrm{Na}$ primeira etapa foram considerados: leitura do título, palavras-chave, resumo e conclusão. Posteriormente, foram excluídos os trabalhos irrelevantes para as questões investigadas.

Dos 70 artigos encontrados com o auxílio da string de busca, 43 foram excluídos por não se encaixarem na pesquisa, devido à duplicação dos artigos, apenas citar Michel Godet, não criam cenários ou são teóricos sem aplicação. A segunda etapa, cujos resultados estão na Tabela 1, os estudos incluídos na etapa anterior foram lidos, e por meio da consideração dos ramos e ferramentas utilizadas, conseguiu-se extrair os dados.

Tabela 1 - Setor e ferramentas dos artigos selecionados 


\begin{tabular}{|c|c|c|}
\hline Periódicos & Ramos & Ferramentas \\
\hline Communications in Computer and Information Science & Rural & MICMAC \\
\hline Electronic Journal of Information Technology in Construction & Industrial & MICMAC \\
\hline \multirow{3}{*}{ Futures } & Nuclear & MICMAC \\
\hline & Estratégico & MICMAC \\
\hline & $\begin{array}{l}\text { Sustentável e } \\
\text { energético }\end{array}$ & MICMAC \\
\hline $\begin{array}{l}\text { International Conference on Intelligent Systems Design and } \\
\text { Aplications }\end{array}$ & Rural & MICMAC \\
\hline International Journal of Global Energy Issues & Energético & MICMAC \\
\hline International Journal of Technology Intelligence and Planning & Estratégico & $\begin{array}{l}\text { MICMAC, MACTOR e } \\
\text { MULTIPOL }\end{array}$ \\
\hline Journal of Decision Systems & Tecnológico & MACTOR \\
\hline Journal of Futures Studies & Saúde & MICMAC \\
\hline Journal of Modelling in Management & Energético & MICMAC \\
\hline Journal of Sustainable Tourism & Turismo & SMIC \\
\hline $\begin{array}{c}\text { Philosophical Transactions of the Royal Society A: Mathematical, } \\
\text { Physical and Engineering Sciences }\end{array}$ & Construção & MICMAC \\
\hline Regional Science Inquiry & Agrícola & $\begin{array}{l}\text { MIC MAC, MACTOR, } \\
\text { SMIC, MORPHOL, } \\
\text { MULTIPOL }\end{array}$ \\
\hline Revista Portuguesa de Saúde Pública & Saúde & MICMAC \\
\hline Sustainability: Science, Practice, and Policy & $\begin{array}{l}\text { Sustentável e } \\
\text { alimentício }\end{array}$ & MICMAC \\
\hline \multirow{4}{*}{ Technological Forecasting and Social Change } & Energético & MICMAC E MACTOR \\
\hline & Setor rural & MICMAC \\
\hline & Planejamento & MICMAC \\
\hline & Nuclear & MICMAC E MACTOR \\
\hline Veterinária Italiana & $\begin{array}{l}\text { Pecuário e } \\
\text { aquicultura }\end{array}$ & MICMAC \\
\hline Environment, Development and Sustainability & Petrolifero & MICMAC \\
\hline European Journal of Futures Research & Educação & SMIC \\
\hline
\end{tabular}

Fonte: Dados da Scopus. Elaborado pelos autores

\subsection{AUTORES COM O MAIOR NÚMERO DE PUBLICAÇÕES}

Segundo a Figura 4, os artigos apontados pela pesquisa são de diferentes autores, avaliando a diversificação sobre o assunto e referência na área da pesquisa. Contudo, autores pioneiros na corrente francesa obtiveram destaque e foram inseridos nesta classificação.

Figura 4 - Artigos por autor 


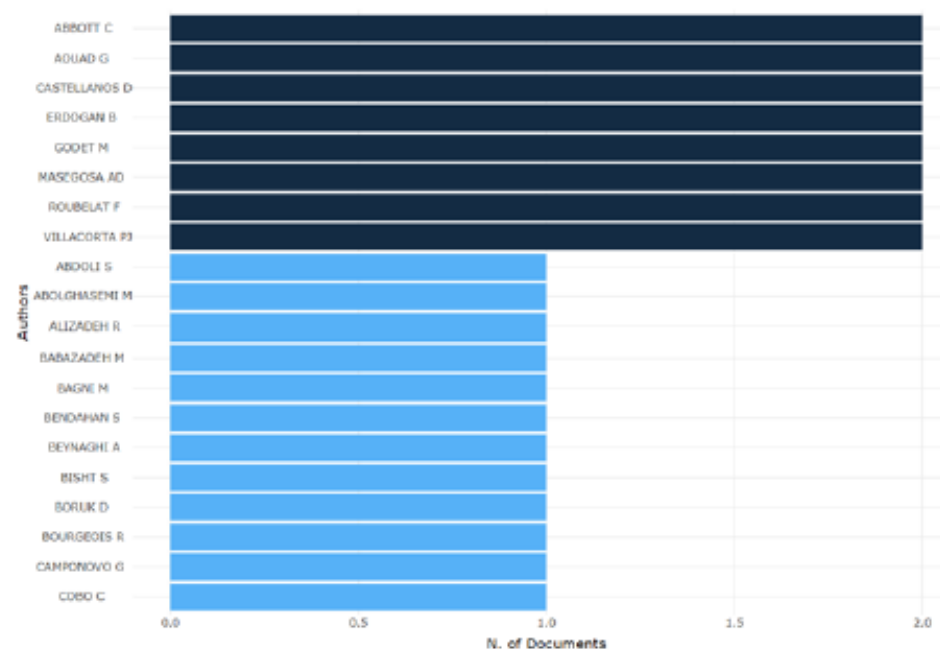

Fonte: Dados da bibliometrix

\subsection{QUANTITATIVO DE ARTIGOS POR ANO}

De acordo com a Figura 5, os artigos presentes na área de cenários prospectivos tiveram início em 1986, porém só a partir de 2000 tornaram-se mais frequentes, tendo seu ápice em 2012 com 3 artigos. A pesquisa foi finalizada em 24 de maio de 2019 e foram identificados 2 artigos para o ano de 2018.

Figura 5 - Artigos por ano

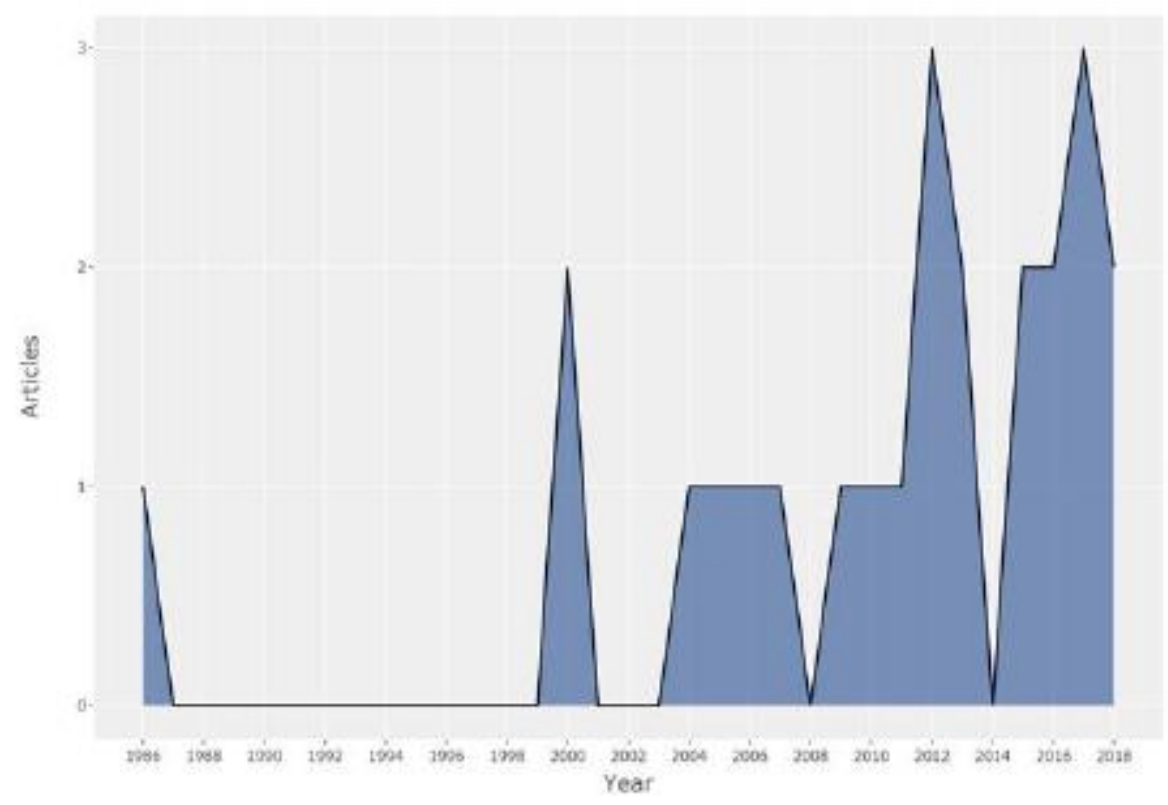

Fonte: Dados da bibliometrix

\subsection{INSTITUIÇÕES MAIS REPRESENTATIVAS}

As 10 instituições com mais destaque estão indexadas na base Scopus, abordando os cenários prospectivos na Figura 6. A Futures e a Technological Forecasting and Social Change possuem 4 artigos que usam cenários prospectivos. As demais instituições da Figura 6, possuem 1 publicações cada, sendo a maioria delas localizadas na Europa. 
Figura 6 - Artigos por instituição

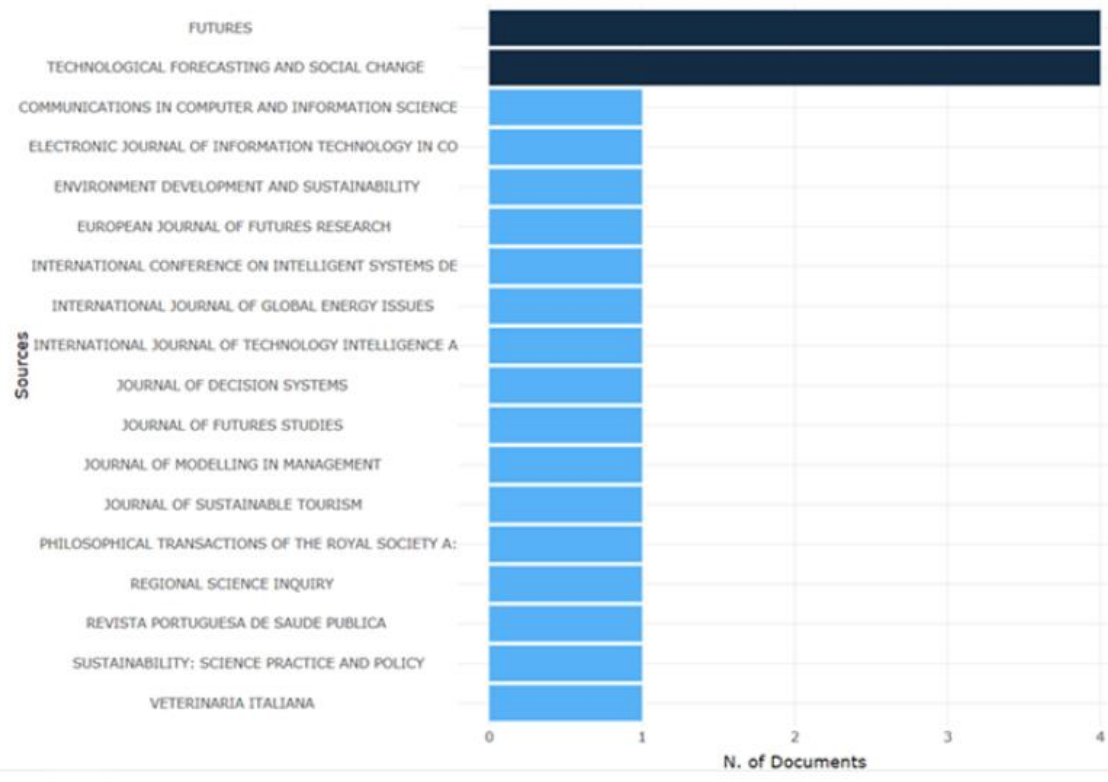

Fonte: Dados da bibliometrix

\subsection{ANÁLISE DE INSTITUIÇÕES MAIS CITADAS}

As instituições mais citadas são representadas na Figura 7. A Futures possui 74 citações, Technological Forecasting and Social Change com 52 citações, Long Range Planning com 17 citações, Foresight com 14 citações, Energy Policy com 13 citações, European Journal of Operational Research com com 12 citações, Harvard Business Review com 9 citações, Pharm World Sci com 7 citações.

Figura 7 - Instituições mais citadas

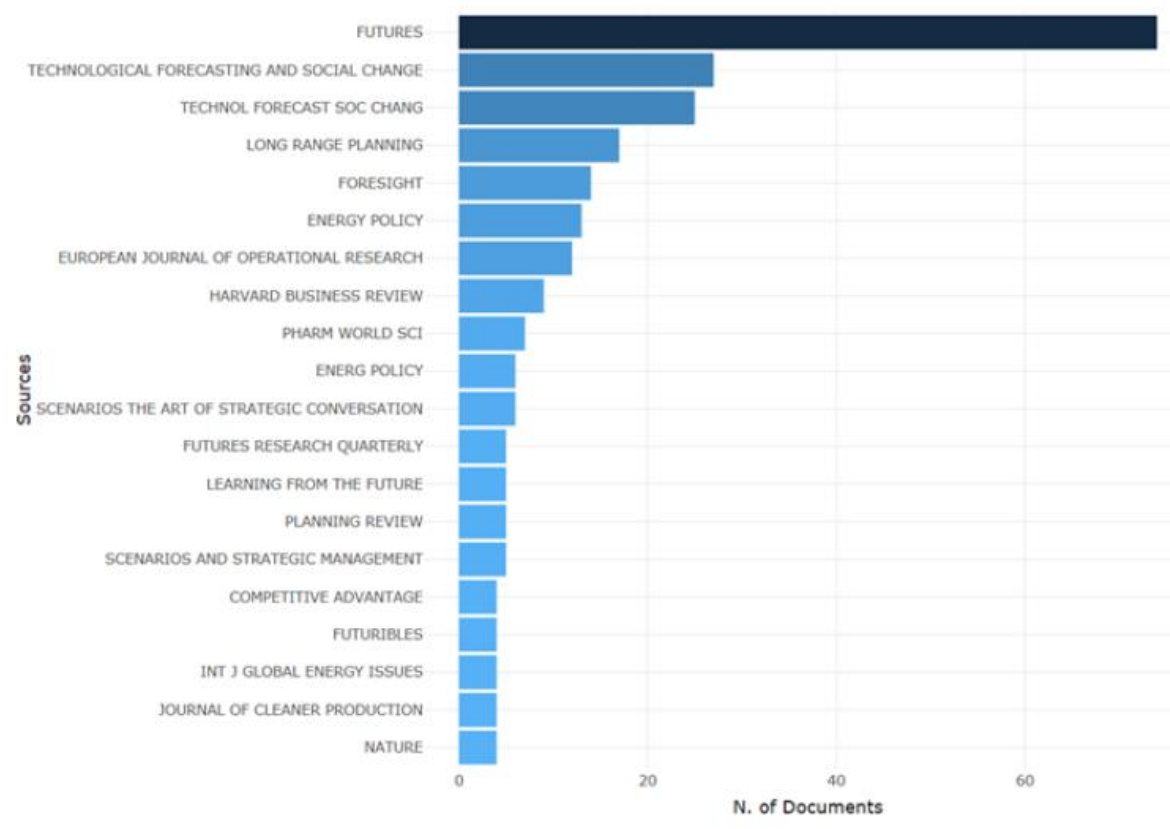

Fonte: Dados da bibliometrix

\subsection{ANÁLISE DOS PAÍSES}

Na figura 8 está representado o quantitativo de artigos por país. 
Figura 8 - Artigos por país

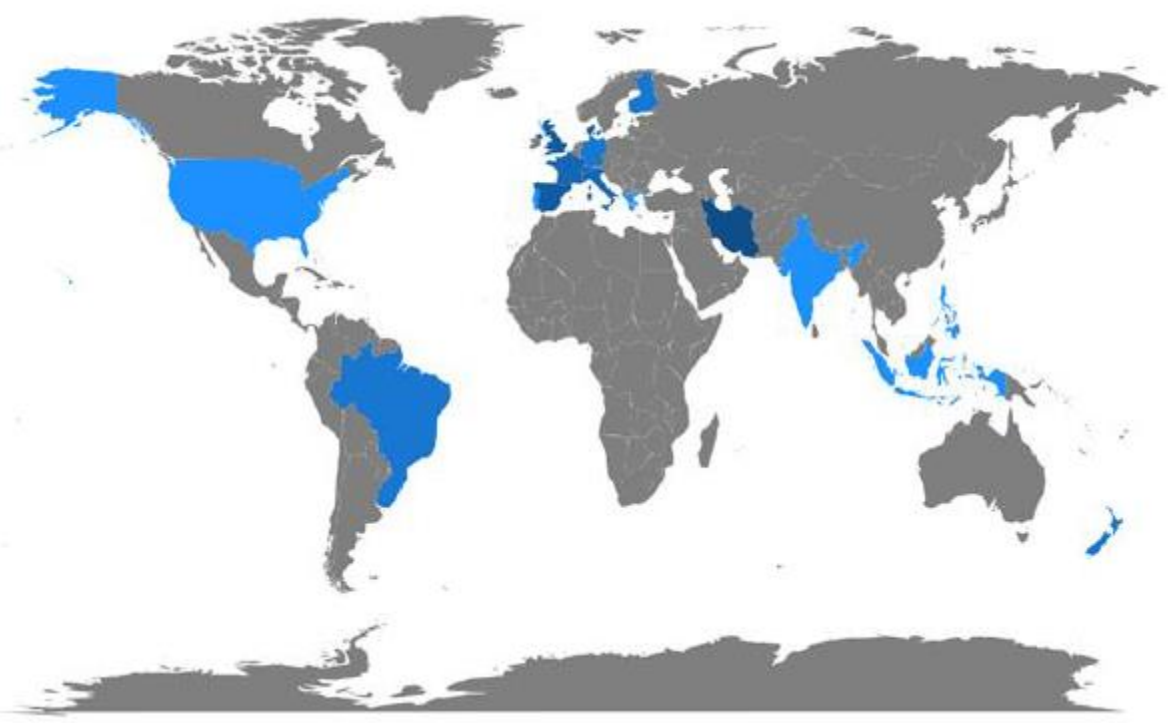

Fonte: R Studio com o pacote biblioshiny

Segundo o mapa apresentado anteriormente, é possível destacar a quantidade de artigos por país resultantes da pesquisa. O Irã apresenta 6 artigos, conforme mostrado com a cor azul escuro, adquirindo destaque diante do restante. Os países que possuem 4 artigos com cor azul médio, são: Reino Unido e Espanha. Os países que possuem 3 artigos são: França e Itália. Os países que possuem 2 artigos são: Alemanha, Brasil, Nova Zelândia e Finlândia. Já os países com 1 artigo são: Indonésia, Filipinas, Índia, EUA, Grécia e Portugal.

\subsection{ANÁLISE DE PAÍSES MAIS CITADOS}

Na Figura 9 mostra-se os países que possuem mais citações no método dos cenários de Michel Godet. É esperado que a França se destaque devido ao seu pioneirismo no $L a$ Prospective.

Figura 9 - Países mais citados

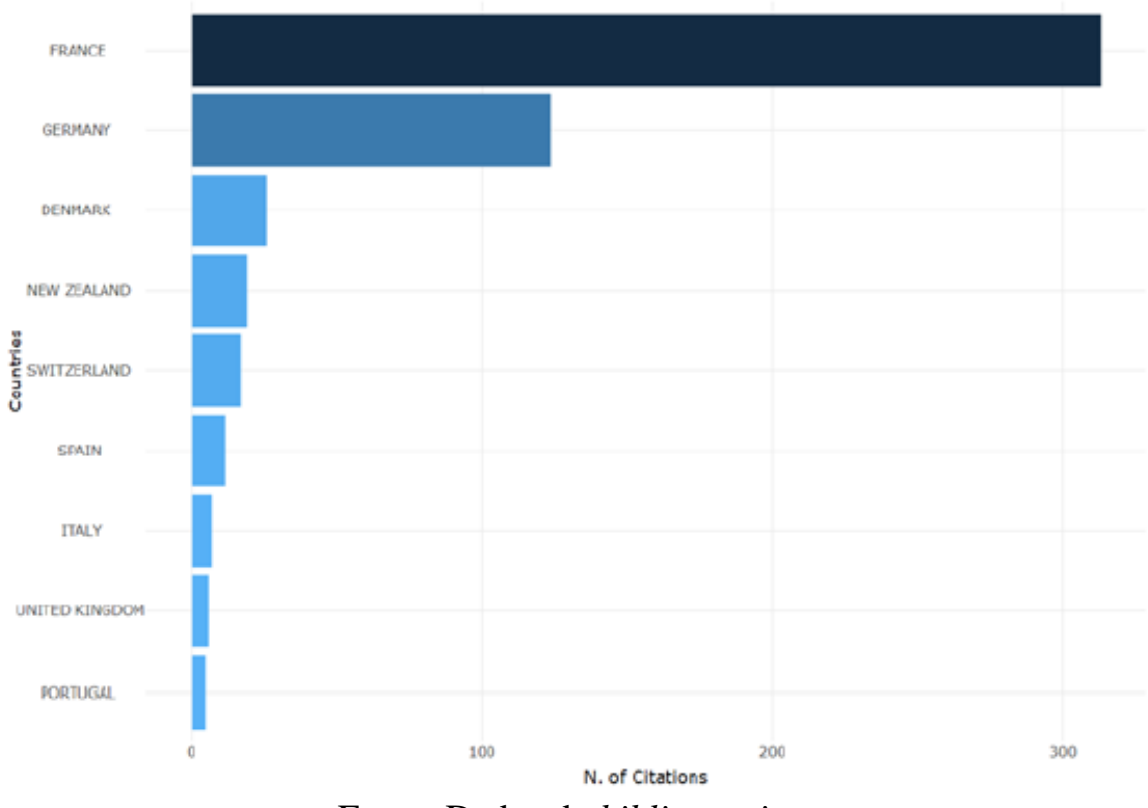

Fonte: Dados da bibliometrix 
A partir dos dados coletados, a França possui 313 citações, a Alemanha com 124 citações, a Dinamarca com 26 citações, Nova Zelândia com 19 citações, a Suíça com 17 citações, a Espanha com 12 citações, Itália com 7 citações, Reino Unido com 6 citações e Portugal com 5 citações.

\subsection{ANÁLISE DAS PALAVRAS-CHAVE}

As palavras-chave dos periódicos resultantes da pesquisa, podem ser observadas na nuvem de palavras da Figura 10. A nuvem de palavras foi formada a partir do uso do software $\mathrm{R}$ com o pacote biblioshiny, permitindo a visualização das palavras proporcionalmente e a frequência em que aparecem na pesquisa.

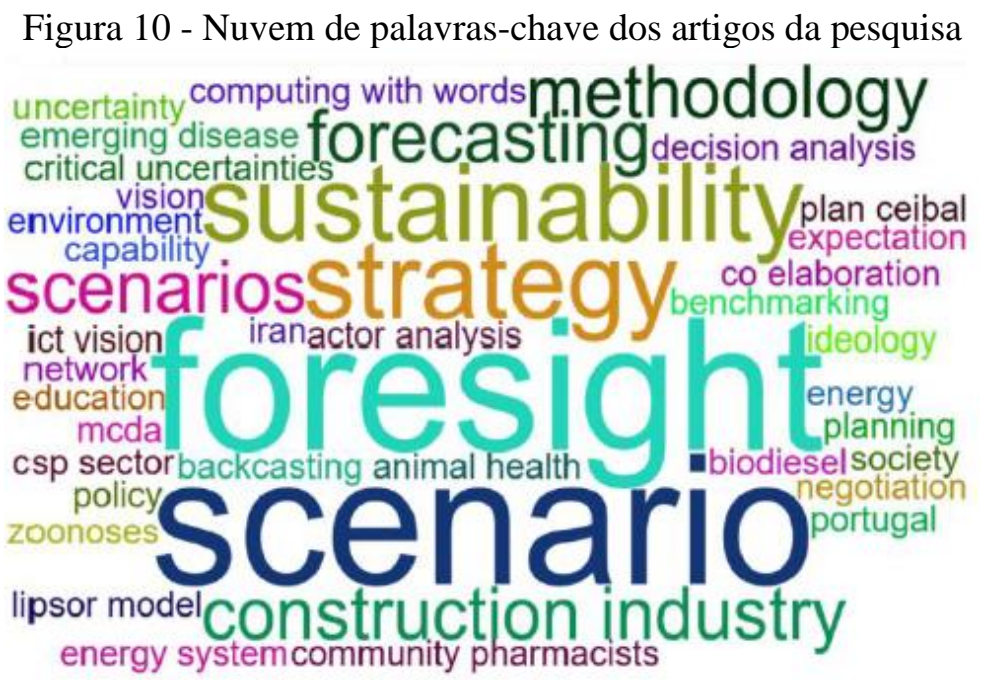

Fonte: Dados da bibliometrix

Pode-se concluir que os artigos, citam em ordem decrescente foresight, scenario, sustainability, strategy, construction industry, forecasting, methodology.

\subsection{ANÁLISE DAS TÍTULO E RESUMO}

As cores indicam clusters de palavras que foram mais fortemente relacionados através das arestas do grafo. Previamente definiram que o termo se repetiria duas vezes e a representação gerada foram quatro clusters (azul, vermelho, amarelo e verde), conforme mostrado na Figura 11. Na Figura 12, cada nó tem uma cor que depende da densidade desse item, ou seja, a cor de um ponto no grafo depende da importância dos itens vizinhos.

Figura 11 - Estrutura colaborativa dos termos no título e resumo

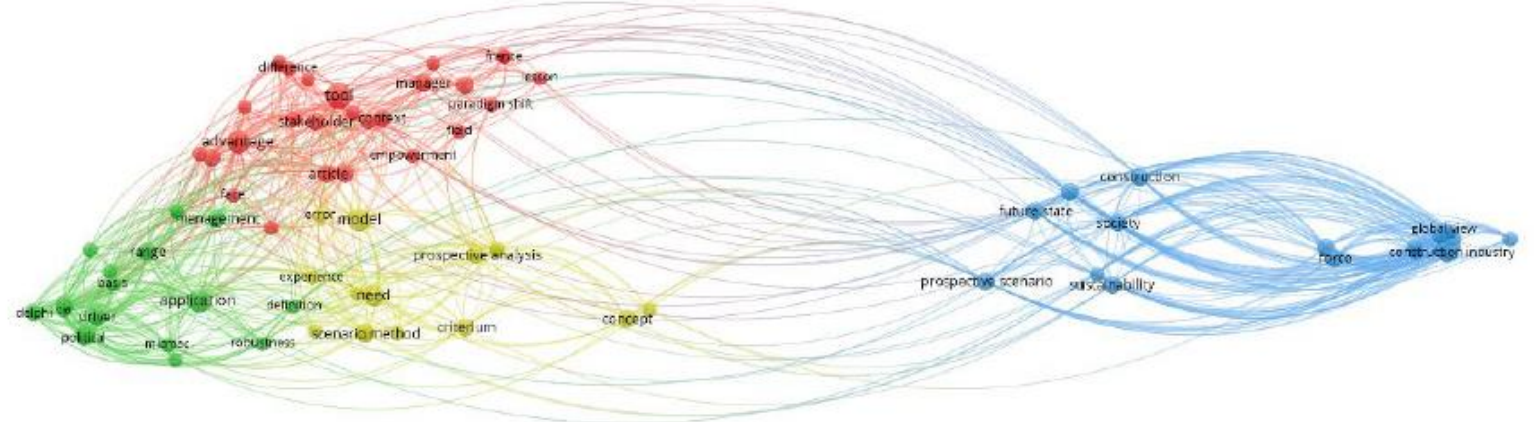

Fonte: VOS Viewer 
Figura 12 - Mapa de calor dos termos no título e resumo

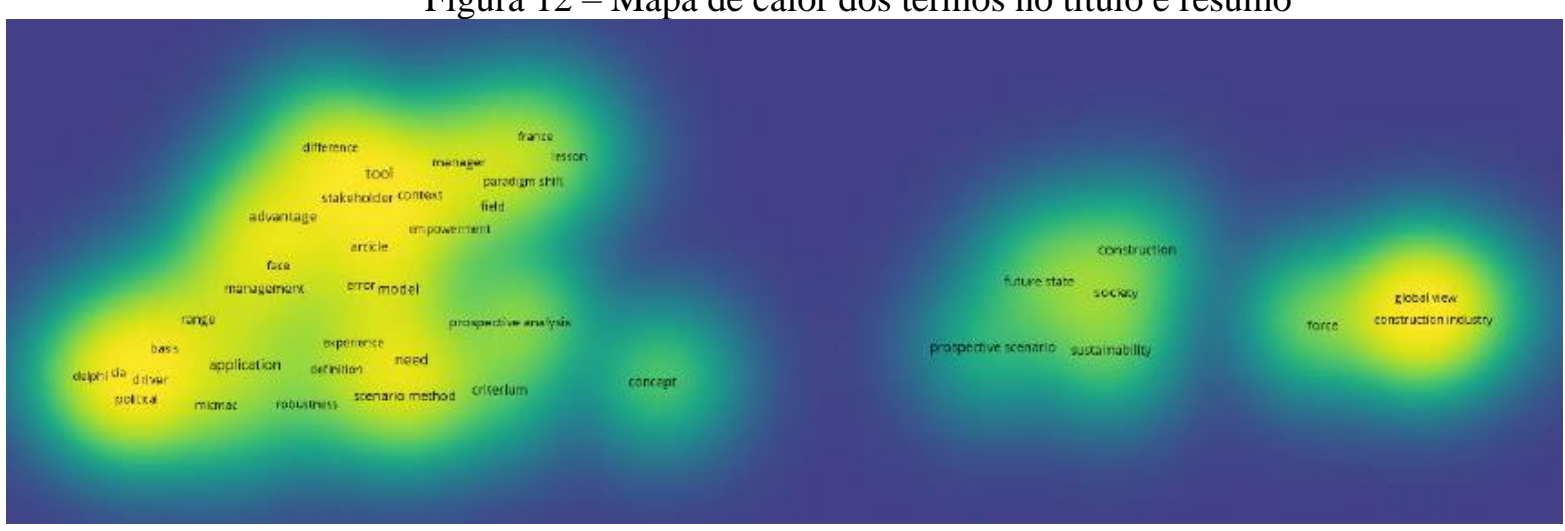

Fonte: VOS Viewer

\section{CONCLUSÕES}

Neste artigo foi realizado um mapeamento sistemático da literatura para a investigação e entendimento do modo que o método dos cenários contribui em diferentes ramos, a partir da utilização das ferramentas apropriadas em diferentes circunstâncias. Os resultados obtidos através da query pesquisada indicam que os artigos não utilizam todas as ferramentas, o que é justificado pela sua adaptação ao aplicar em diferentes casos, com o intuito de alcançar uma abrangência consistente.

O objetivo proposto foi atendido ao apresentar um estudo compilando quais anos, autores, instituições, países, áreas, ferramentas e palavras-chave estão mais relacionados com o método dos cenários e como este alcançou destaque acadêmico, segundo as informações extraídas na base Scopus. No entanto, foi feito uma pesquisa completa ao analisar os países e instituições mais citados e a relação entre as palavras chaves relevantes no resumo e título a fim de confirmar as fortes relações entre os artigos.

O Software $\mathrm{R}$ em conjunto com as bibliotecas bibliometrix e biblioshiny, mostraram-se eficientes na extração de resultados, pois foi gerado gráficos e figuras de modo rápido. Portanto, simplificou-se a coleta de dados que é uma tarefa penosa, além da disponibilidade gratuita e de código aberto.

Como trabalhos futuros, pretende-se ampliar o mapeamento sistemático na busca de aplicar esta abordagem em empresas de determinados ramos com o uso da ferramenta apropriada a fim de enaltecer a criação de estratégias para cenários futuros. Portanto, irá influenciar no uso em pesquisas no meio científico e servir de apoio para situações semelhantes, no qual indicará as ferramentas adequadas e como foi aplicada para a criação de cenários.

As sugestões para os próximos trabalhos são um estudo acrescentando outras bases de dados como Science Direct, Web of Science para fornecer outras informações complementares e ampliar o conhecimento diante das ferramentas usadas em cenários prospectivos. Devido a limitação do estudo, não é possível classificar comparado com outras bases, visto que foi considerada apenas a Scopus.

\section{REFERÊNCIAS BIBLIOGRÁFICAS}

[1] ARIA, M.; CUCCURULLO C. Bibliometrix: An R-tool for comprehensive science mapping analysis, Journal of Informetrics, 11(4), pp 959-975, Elsevier, 2017.

[2] AZEVEDO FILHO, Edson Terra; PERESTRELO, Margarida; MOLINA-PALMA, Manuel António. As descobertas do pré-sal e os desafios competitivos da indústria brasileira do setor de petróleo e gás: Uma abordagem prospectiva. CIDADES, 2015. 
[3] CHADEGANI, Arezoo Aghaei; SALEHI, Hadi; YUNUS, Melor; EBRAHIM, Nader Ale. A Comparison between Two Main Academic Literature Collections: Web of Science and Scopus Databases. Asian Social Science, 2013.

[4] CUCCURULlO, C.; ARIA, M.; SARTO, F. Foundations and trends in performance management. A twenty-five years bibliometric analysis in business and public administration domains, Scientometrics, 2016.

[5] DIXON-WOODS, M., AGARWAL, S., JONES, D., YOUNG, B. \& SUTTON, A., Synthesising qualitative and quantitative evidence: a review of possible methods, Journal of Health Services Research and Policy 10, 45-53, 2005.

[6] GODET, Michel. From Anticipation to Action: a Handbook of Stratégie Prospective. Unesco Publishing, 1991.

[7] GODET, Michel. Manual de prospectiva estratégica: da antecipação à acção. Lisboa: Dom Quixote, 1993.

[8] GODET, Michel; DURANCE, Philippe; DIAS, Júlio. (2008). A prospectiva estratégica para as empresas e os territórios, 2008.

[9] GULER, Arzu Tugce; WAAIJER, Catheliin J. F.; PALMBLAD, Magnus. Scientific workflows for bibliometrics. Scientometrics, 2016.

[10] JOSHI, Aditi. Comparison Between Scopus \& Isi Web of Science. Journal Global Values. 7, 2016.

[11] KITCHENHAM, Barbara; CHARTERS Stuart. Guidelines for performing Systematic Literature Reviews in Software Engineering. EBSE Technical Report. Vol 2.3, 2007.

[12] KITCHENHAM, Barbara; BRERETON, Pearl; BUDGEN, David; TURNER, Mark; BAILEY, John; LINKMAN, Stephen. Protocol for a Tertiary Study of Systematic Literature Reviews and Evidence-based Guidelines in IT and Software Engineering, 2007.

[12] PETERSEN, Kai; FELDT, Robert; MUJTABA, Shahid; MATTSSON, Michael. Systematic Mapping Studies in Software Engineering. 12th International Conference on Evaluation and Assessment in Software Engineering, 2008.

[13] PETERSEN, Kai; VAKKALANKA, Sairam; KUZNIARZ, Ludwik. Guidelines for conducting systematic mapping studies in software engineering: An update. Information and Software Technology, Volume 64, 2015, p. 1-18, 2015

[14] PORTER, Michael E. Competitive Strategy. New York: Free Press, 1985.

[15] RODRIGUES, A.; ROJO, C. A.; BERTOLINI, G. R. F. Formulação de estratégias competitivas por meio de análise de cenários na construção civil. Produção, v. 23, n. 2, p. 269282, abr./jun, 2013.

[16] SARAGOÇA, J. Governo Electrónico Local: Diagnóstico Sociológico, Estratégias de Actores e Futuros Possíveis para o Distrito de Évora, Portugal. Diagnóstico e Prospetiva Social. Évora: Universidade de Évora, 2012.

[17] SCHÜTZ, Gustavo Ricardo; SANT'ANA, Antônio Sérgio; SANTOS, Saray Giovana. Política de periódicos nacionais em educação física para estudos de revisão sistemática. Revista Brasileira de Cineantropometria do Desempenho Humano, Santa Catarina, v. 13, n. 4, p. 313319. 2011.

[18] SOUZA, Juliana Bittar. Planejamento de Cenários Prospectivos: Um breve debate sobre as abordagens existentes. XII SEMEAD- Seminários em Administração, São Paulo. XII SEMEAD, 2009.

[19] SUTTER, Mariana Bassi; ESTIMA, Daniel; POLO, Edison Fernandes; COULTER, James Terence. Construção de Cenários: Apreciação de Métodos mais utilizados na Administração Estratégica. Espacios, Vol. 33 (8) 2012. p. 13, 2012. 